\title{
The Stellar Content of NGC 6789, A Blue Compact Dwarf Galaxy in the Local Void $^{1}$
}

\author{
Igor O. Drozdovsky \\ University of Pittsburgh, Pittsburgh, PA 15260, USA, and Astronomical Institute, St.Petersburg \\ State University, Russia \\ dio@phyast.pitt.edu \\ Regina E. Schulte-Ladbeck \\ University of Pittsburgh, Pittsburgh, PA 15260, USA \\ rsl@phyast.pitt.edu \\ Ulrich Hopp \\ Universitätssternwarte München, München, FRG \\ hopp@usm. uni-muenchen.de \\ Mary M. Crone \\ Skidmore College, Saratoga Springs, NY 12866, USA \\ mcrone@skidmore.edu \\ Laura Greggio \\ Osservatorio Astronomico di Bologna, Bologna, Italy, and Universitätssternwarte München, \\ München, FRG \\ greggio@usm.uni-muenchen.de
}

October 31, 2018

\begin{abstract}
We find that NGC 6789 is the most nearby example of a Blue Compact Dwarf galaxy known to date. With the help of WFPC2 aboard the Hubble Space Telescope, we resolve NGC 6789 into over 15,000 point sources in the V and I bands. The young
\end{abstract}

\footnotetext{
${ }^{1}$ Based on observations made with the NASA/ESA Hubble Space Telescope obtained from the Space Telescope Science Institute, which is operated by the Association of Universities for Research in Astronomy, Inc., under NASA contract NAS 5-26555.
} 
stars of NGC 6789 are found exclusively near the center of the galaxy. The red giant population identified at large galacticentric radii yields a distance of about $3.6 \mathrm{Mpc}$, a stellar metallicity $[\mathrm{Fe} / \mathrm{H}]$ of about -2 , and a minimum age of about 1 Gyr. Despite its isolated location in the Local Void, its low metallicity, and its active star formation, the properties of NGC 6789 are clearly not those of a galaxy in formation.

Subject headings: Galaxies: compact — galaxies: dwarf — galaxies: evolution — galaxies: individual (NGC 6789) — galaxies: stellar content — galaxies: distances and redshifts

\section{Introduction}

Galaxies in voids are interesting for their potential use as probes of Cold Dark Matter (CDM) cosmogonies (Dekel \& Silk 1986). In general, these models predict that isolated galaxies are less evolved than those in denser environments. For example, N-body simulations show that dark matter halos in lower-density regions tend to have lower masses (e.g. Lemson \& Kauffmann 1999), making them more susceptible to negative feedback from ionizing radiation and stellar winds (Ferrara \& Tolstoy 2000). Indeed, recent high-resolution simulations which incorporate a semi-analytic prescription for star formation specifically predict that star formation is inhibited in voids (White \& Springel 2000). Clearly, these models should be tested against the observed star-formation history of void galaxies.

The most straightforward way to observe the star-formation history of galaxies is through deep single-star photometry, which can directly identify stars in various evolutionary phases through their positions on a color-magnitude diagram (CMD). While observations of chemical abundances can be performed for more distant star-forming galaxies, the validity of age-dating from certain element abundance ratios has been challenged by recent chemical evolution models, which indicate that even extremely low-metallicity galaxies can have formed many Gyr ago (Henry, Edmunds \& Köppen 2000).

When Karachentsev \& Makarov (1998) presented NGC 6789 as an unevolved dwarf galaxy (akin to NGC 1705 and NGC 2915, e.g. Meurer et al. 1992) in the direction of the Local Void in Hercules-Aquila (with a heliocentric velocity of $-147 \mathrm{~km} \mathrm{~s}^{-1}$ ), we had found the perfect candidate for a stellar population study of a low-mass void galaxy with the HST. NGC 6789 is extremely isolated (according to a NED query with a $40^{\circ}$ search radius and Karachentsev, private communication); it is separated by at least $2.5 \mathrm{Mpc}$ from the massive galaxy NGC 6946. NGC 6789 has the properties of Blue Compact Dwarfs (BCDs), systems long considered candidates for young galaxies in the nearby Universe (Sargent \& Searle 1970).

Meanwhile, Drozdovsky \& Tikhonov (2000, hereafter DT) imaged NGC 6789 from the ground. They found that it belongs to the iE subtype, which exhibits the morphology most characteristic 
of BCDs. This result, together with our work on the distribution of red giants in the $\mathrm{iE} \mathrm{BCD}$ VII Zw 403 (Schulte-Ladbeck et al. 1999), already suggests the presence of stellar generations which pre-date the on-going starburst. DT identified the faint red stars in their CMD with the tip of the red giant branch (TRGB), and derived a very short distance of only 2.1 Mpc (m-M=26.6). However, as our HST data show, DT identified bright asymptotic giant branch (AGB) stars with the TRGB, severely underestimating the true distance of NGC 6789. Nevertheless, in this paper we show that NGC 6789 is still the most nearby BCD on record; and the presence of RGB stars allows us to ascertain that it is at least about 1 Gyr old.

\section{Observations and reductions}

Observations of NGC 6789 were obtained July through September of 2000 with the WFPC2 aboard HST as part of GO programs 8645 and 8122. In Fig. 1, we overlay deep WFPC2 photometry on the Nordic Optical Telescope (NOT) image of DT. The central star-forming region was encompassed by the PC chip, whereas the WF chips were pointed into the low-surface-brightness

sheet. Resolved halo stars can be traced to large distances from the center of NGC 6789 (see the physical scale of Fig. 1).

We discuss photometry based on dithered exposures in the F555W (V) and F814W (I) bands taken as part of our program 8122. We performed the usual post-pipeline reductions and used drizzling to combine individual exposures. The total exposure times are $8,200 \mathrm{~s}$ each in V and I. Fig. 2 shows the color image of the PC, exhibiting many resolved stars. We performed singlestar photometry with DAOPHOT II (Stetson 1994). The WF chips have a low stellar density ( $\sim 0.4$ stars $/ \square^{\prime \prime}$; showing many background galaxies which were rejected in the photometry); and we could easily find isolated stars to model the point-spread function (PSF). On the contrary, the PC chip has a very high stellar density $\left(\sim 6.7\right.$ stars $\left./ \square^{\prime \prime}\right)$, much higher than what we encountered in our analysis of UGCA $290\left(\sim 2.0\right.$ stars/ $\square^{\prime \prime}$; Crone et al. 2000) or VII Zw $403\left(\sim 2.8\right.$ stars $/ \square^{\prime \prime}$; Schulte-Ladbeck et al. 1999). It was difficult to find suitable stars to model the PSF, and these data are clearly affected by blending due to crowding in a substantial way. This leads to a shallow detection limit for the PC data (see Fig. 3a). We took zero points from the May 1997 SYNPHOT tables, and corrected for foreground extinction in the direction of NGC 6789 using the values of Schlegel, Finkbeiner \& Davis (1998) with $\mathrm{R}_{V}=3.1: \mathrm{A}_{V}=0.232$ and $\mathrm{A}_{I}=0.136$. We did not correct for internal extinction within NGC 6789, but the transparency of the halo population (the population from which we derive the distance) is high and suggests that the extinction is low at high galacticentric radii. We performed ADDSTAR tests on the WF chips and found that completeness is high $(>90 \%)$ well below the TRGB. 


\section{Results}

The CMDs of NGC 6789 illustrate the spatial variation in its stellar content from the "core" to the "halo". The CMD of the PC (Fig. 3a) exhibits a stellar mix which is characteristic of the superposition of many stellar generations. The "blue plume" at $(\mathrm{V}-\mathrm{I}) \sim 0$ contains massive blue supergiants and main-sequence stars, the "red plume" at $(\mathrm{V}-\mathrm{I}) \sim 1.5$ contains evolved supergiants and AGB stars, the "red tail" extending past $(\mathrm{V}-\mathrm{I})>2$ contains intermediate-mass AGB stars in the thermally pulsing phase, and the concentration of stars below the TRGB contains low-mass red giants and AGB stars. For reference, we overlay the CMD with the $\mathrm{Z}=0.0004$ and 0.004 Padova stellar evolutionary tracks (Fagotto et al. 1994) assuming the distance derived below. The blue plume is quite wide, and the TRGB looks slightly brighter than tip of $1 \mathrm{M}_{\odot}$ tracks. Artificial star tests show that crowding and blending dominate these effects, while unknown internal extinction variations may also contribute.

The WF chips lack blue stars down to the detection limit (see Fig. 3b). This suggests that the average population age is higher in the halo than it is in the core. The CMD of the halo is characterized by a comparatively narrow red giant branch. We overplot onto the CMD a few Globular Cluster ridgelines for low-metallicity clusters from Da Costa \& Armandroff (1990). Additional stars scattered across the CMD are attributed to galactic foreground contamination: the galactic latitude of NGC 6789 is only 21.5 (and see Fig. 1).

We used the WF data to find the TRGB, and to determine the distance and metallicity of NGC 6789 (following Lee, Freedman \& Madore 1993). The WF data are preferred over the PC data since they exhibit much less crowding, and fewer AGB stars. There are sufficiently large numbers of stars found on each chip, that we were able to construct I-band luminosity functions for each chip individually. The location of the TRGB occurs at the same magnitude in all three chips. The final TRGB magnitude was derived from the combined I-band luminosity function of about 7,000 stars selected to have $0.7<(\mathrm{V}-\mathrm{I})_{0}<2.0$ (see Fig. 4). The TRGB was found by applying a Sobel filter; it occurs at $\mathrm{I}_{0}=23.82 \pm 0.05$. In order to derive the metallicity of the RGB stars we measure the $(\mathrm{V}-\mathrm{I})_{0}$ color both at half a magnitude below the TRGB and, owing to the high quality of the data, also at one magnitude below the TRGB. We consistently find a low metallicity, which, at 0.5 mag below the TRGB, is $[\mathrm{Fe} / \mathrm{H}]=-1.92 \pm 0.03 \pm 0.15$, where the first error is the uncertainty in the mean and the second is the systematic error. With the appropriate bolometric correction, the absolute magnitude of the TRGB is -4.0 , yielding a distance modulus of $27^{\mathrm{m}} \cdot 80 \pm 0.13 \pm 0.18$, or a distance of $3.6 \pm 0.2 \pm 0.3 \mathrm{Mpc}$. This location of the TRGB is indicated in Fig. 3a and 3b by a dashed line.

NGC 6789 is about 70\% more distant than was derived by DT. At this new distance, an angular scale of $1^{\prime}$ corresponds to a physical scale of $1.05 \mathrm{kpc}$. The star-formation rate derived by DT from the $\mathrm{H}_{\alpha}$ luminosity increases to $0.04 \mathrm{M}_{\odot} \mathrm{yr}^{-1}$ (for a Salpeter initial mass function ranging from 0.1-100 $\mathrm{M}_{\odot}$, i.e., Hunter \& Gallagher 1986). The integrated total magnitudes of NGC 6789 within the $\mu_{\mathrm{V}}=25 \mathrm{mag} / \square^{\prime \prime}$ isophote of DT, $m_{\mathrm{I}}=13.63 \pm 0 .{ }^{\mathrm{m}} 15$ and $m_{\mathrm{V}}=14^{\mathrm{m}} \cdot 62 \pm 0 .{ }^{\mathrm{m}} 15$, correspond 
to absolute magnitudes of $M_{\mathrm{I}, 0}=-14^{\mathrm{m}} 31 \pm 0.20$ and $M_{\mathrm{V}, 0}=-13^{\mathrm{m}} 41 \pm 0.20$. DT show that the outer regions of NGC 6789 exhibit the exponential surface brightness profile of a disk galaxy. The scale length correponds to 280 pc, much smaller than that of VII Zw 403 ( $\approx 600$ pc, Schulte-Ladbeck et al. 1999). S. Moehler kindly obtained a spectrum of NGC 6789 for us from which we measured the strengths of [OII], [OIII], [NII] and H-Balmer emission lines. The temperature-sensitive [OIII] line at $4636 \AA$ is undetected. This leaves $12+\log (\mathrm{O} / \mathrm{H})$ two-valued (Edmunds \& Pagel 1984) at 7.7 or 8.5. While NGC 6789 appears to have a sub-solar oxygen abundance, its N/O ratio (estimated following Thurston, Edmunds \& Henry 1996) is larger than the minimum value seen in BCDs, $\log (\mathrm{N} / \mathrm{O})=-1.6$ (Izotov \& Thuan 1999). Therefore, NGC 6789 is probably not one of the rare extremely metal-poor BCDs resembling I Zw 18, but rather, a BCD with moderately low ionized gas metallicity.

NGC 6789 is undetected in HI (Huchtmeier et al. 2000). Possible reasons given are confusion with local Hi or lack of sensitivity for very weak emission. If NGC 6789 could indeed be shown to possess very little neutral gas, it could be interpreted as a BCD near the end of its life.

\section{Discussion}

We start by comparing the properties of NGC 6789 to those of the other four BCDs which are resolved with HST to magnitudes below the TRGB. NGC 6789, at a mere $3.6 \mathrm{Mpc}$, is the most nearby BCD resolved to date. Ordered by increasing distance we have: Mrk $178(\geq 4.2 \pm 0.5 \mathrm{Mpc}$,

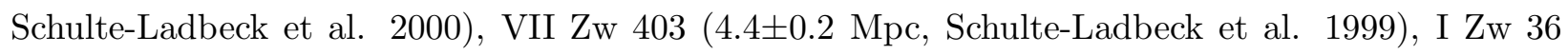
( $\geq 5.8 \pm 0.4 \mathrm{Mpc}$, Schulte-Ladbeck et al. 2001), and UGCA 290 (6.7 \pm 0.4 Mpc, Crone et al. 2000). NGC 6789, situated in the Local Void, is the BCD in the most underdense region. VII Zw 403 is on the line of sight to M 81 but at least a Mpc behind the center of the group. Mrk 178, I Zw 36 and UGCA 290 all lie in the direction of the Canes Venatici Cloud.

The stellar population in NGC 6789 exhibits several features typical of the other resolved BCDs. For example, the CMD of the star-forming core of NGC 6789 exhibits a prominent population of luminous AGB stars. This serves to indicate that star formation in the centers of BCDs (at the bottom of the gravitational well) has continued to be active over the past several hundred Myr. All of the nearby BCDs resolve into red giants, which in all but UGCA 290, are much more extended spatially than the young, main sequence and AGB stars. We have termed these halos "Baade's red sheets" (Schulte-Ladbeck, Crone \& Hopp 1998). Because stars of a wide range of ages overlap on the RGB, we can only be sure that these stars are at least 1 Gyr old, while they could in principle be as old as the oldest stars in the Universe. Whenever optical photometry is available we find that the halo populations of BCDs exhibit blue RGBs, which can be interpreted to indicate low mean

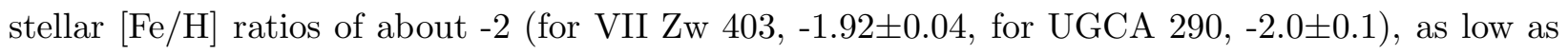
those of the most metal poor galactic Globular Clusters.

The current star-formation rate of NGC 6789 is not particularly high. The rates are never 
exceptionally high in the cases we have investigated, only of order a few times $10^{-3}-10^{-2} \mathrm{M}_{\odot} \mathrm{yr}^{-1}$,

from either or both, $\mathrm{H}_{\alpha}$ luminosity and CMD modeling. They are orders of magnitude below the high rates, $10^{0}-10^{2} \mathrm{M}_{\odot} \mathrm{yr}^{-1}$, determined for a sample of 27 BCDs in the Second Byurakan Survey (Izotova, Parnovsky \& Izotov 2000).

The question of whether BCDs in voids are statistically different from those in the field is an area of active research. Vílchez (1995) compared BCDs in the Virgo cluster with isolated BCDs. He found that BCDs in low density environments are likely to exhibit their first major episode of star formation. More recently, however, Popescu, Hopp \& Rosa (1999) investigated a sample of BCDs in voids and in the field, and found no differences between their star-formation rates or metallicities. Moreover, Vennik, Hopp, \& Popescu (2000) performed B and R surface photometry on the above galaxy sample, and found that field and void BCDs are indistinguishable in terms of luminosity and color. They also noted that more than half of their void BCDs show indications for low surface-brightness red disks, possibly due to an older stellar population.

Our results for the most nearby BCDs support the idea that void and field BCDs are similar, in terms of resolved stellar content, star-formation rate, $\mathrm{Fe} / \mathrm{H}$ ratio, and morphology. In particular, they support the hypothesis that void BCDs have stellar background sheets that pre-date the on-going starburst.

The ubiquity of Baade's sheet in every galaxy resolved deeply enough to detect red giants including isolated and low-metallicity star-forming dwarfs — imposes significant constraints on models of galaxy formation and chemical evolution.

Work on this project was supported through HST grants to RSL and MMC (project 8122). UH acknowledges financial support from SFB 375. LG acknowledges support from the Alexander von Humboldt Stiftung. We thank S. Moehler for providing us with her NGC 6789 spectrum. We made extensive use of the SIMBAD and NED data bases.

\section{REFERENCES}

Crone, M., Schulte-Ladbeck, R.E., Hopp, U., \& Greggio, L., 2000, ApJL, 545, 31

Da Costa, G.S., \& Armandroff, T.E. 1990, AJ, 100, 162

Dekel, A., Silk, J. 1986, ApJ, 303, 39

Drozdovsky, I., \& Tikhonov, N. 2000, A\&AS, 142, 347 (DT)

Ferrara, A., \& Tolstoy, E. 2000, MNRAS, 313, 291

Fagotto, F., Bressan, A., Bertelli, G., Chiosi, C. 1994, A\&AS, 104, 365

Edmunds, M.G., Pagel 1984, MNRAS, 211, 507

Henry, R.B.C., Edmunds, M.G., Köppen, J. 2000, ApJ, 541, 660 
Huchtmeier, W.K., Karachentsev, I.D., Karachentseva, V.E., \& Ehle, M. 2000, A\&AS, 141, 469

Hunter, D. A., Gallagher, J. S. 1986, PASP, 98, 5

Izotov, Y.I., Thuan, T.X. 1999, ApJ, 511, 639

Izotova, Parnovskya, S., \& Izotov, Y. 2000, NewAR, 44, 283

Karachentsev, I.D., \& Makarov, D.I. 1998, A\&A, 331, 891

Lee, M.G., Freedman, W.L., \& Madore, B.F., 1993, ApJ, 417, 553

Lemson, G., \& Kauffmann, G. 1999, MNRAS, 302, 111

Meurer, G.R., Freeman, K. C., Dopita, M.A., \& Cacciari, C., 1992, AJ, 103, 60

Popescu, C.C, Hopp, U., \& Rosa, M.R. 1999, A\&A, 350, 414

Sargent, W.L.W., \& Searle, L. 1970, ApJL, 162, 155

Schlegel, D.J., Finkbeiner, D.P., \& Davis, M. 1998, ApJ, 500, 525

Schulte-Ladbeck, R.E., Crone, M.M., \& Hopp, U. 1998, ApJ, 493, L23

Schulte-Ladbeck, R.E., Hopp, U., Crone, M.M. \& Greggio, L. 1999, ApJ, 525, 709

Schulte-Ladbeck, R.E., Hopp, U., Greggio, L., \& Crone, M.M. 2000, AJ, 120, 1713

Schulte-Ladbeck, R.E., Hopp, U., Greggio, L., Crone, M.M., \& Drozdovsky, I.O., 2001, AJ, submitted

Stetson, P.B. 1994, PASP, 106, 250

Thurston, T.R., Edmunds, M.G., \& Henry, R.B.C., 1996, MNRAS, 283, 990

Vennik, J., Hopp, U., \& Popescu C.C., 2000, A\&AS, 142, 399

Vílchez, J.M. 1995, AJ, 110, 1090

White, S.D.M., \& Springel, V. 2000, in "The First Stars", ESO Astrophysics Symposia, eds. A. Weiss, T. Abel, \& V. Hill (Springer), 327 
Fig. 1. - A color picture of the WFPC2-resolved stars from program 8122 overlayed onto the NOT ground-based image of DT. $\mathrm{N}$ is up and $\mathrm{E}$ is to the left. The size of the image is $3.7 \times 3 ! 7$, which corresponds to $\sim 4 \times 4 \mathrm{kpc}$. Notice how far out the resolved stars can be traced.

Fig. 2.- A color image of the PC chip, illustrating the compact, dense core of star formation. The color combinations used are: red $=\mathrm{F} 814 \mathrm{~W}$, green $=\mathrm{F} 555 \mathrm{~W}+\mathrm{F} 814 \mathrm{~W}+\mathrm{F} 656 \mathrm{~N}$, and blue $=$ F555W+F336N.

Fig. 3.- a) The CMD of the PC chip with a range of stellar tracks overlayed for two metallicities. Tracks with metallicities $\mathrm{Z}=0.004$ and 0.0004 are marked by red and green colors respectively. b) The CMD of the WF chips with low Fe/H Globular Cluster ridge lines overlayed. The dashed line on both images indicates the position of the TRGB.

Fig. 4.- The I-band luminosity function (solid line), and the Sobel-filtered luminosity function (dotted line). The location of the TRGB is indicated. 
This figure "fig1.jpg" is available in "jpg" format from: http://arxiv.org/ps/astro-ph/0102452v1 
This figure "fig2.jpg" is available in "jpg" format from: http://arxiv.org/ps/astro-ph/0102452v1 
This figure "fig3a.jpg" is available in "jpg" format from: http://arxiv.org/ps/astro-ph/0102452v1 
This figure "fig3b.jpg" is available in "jpg" format from: http://arxiv.org/ps/astro-ph/0102452v1 


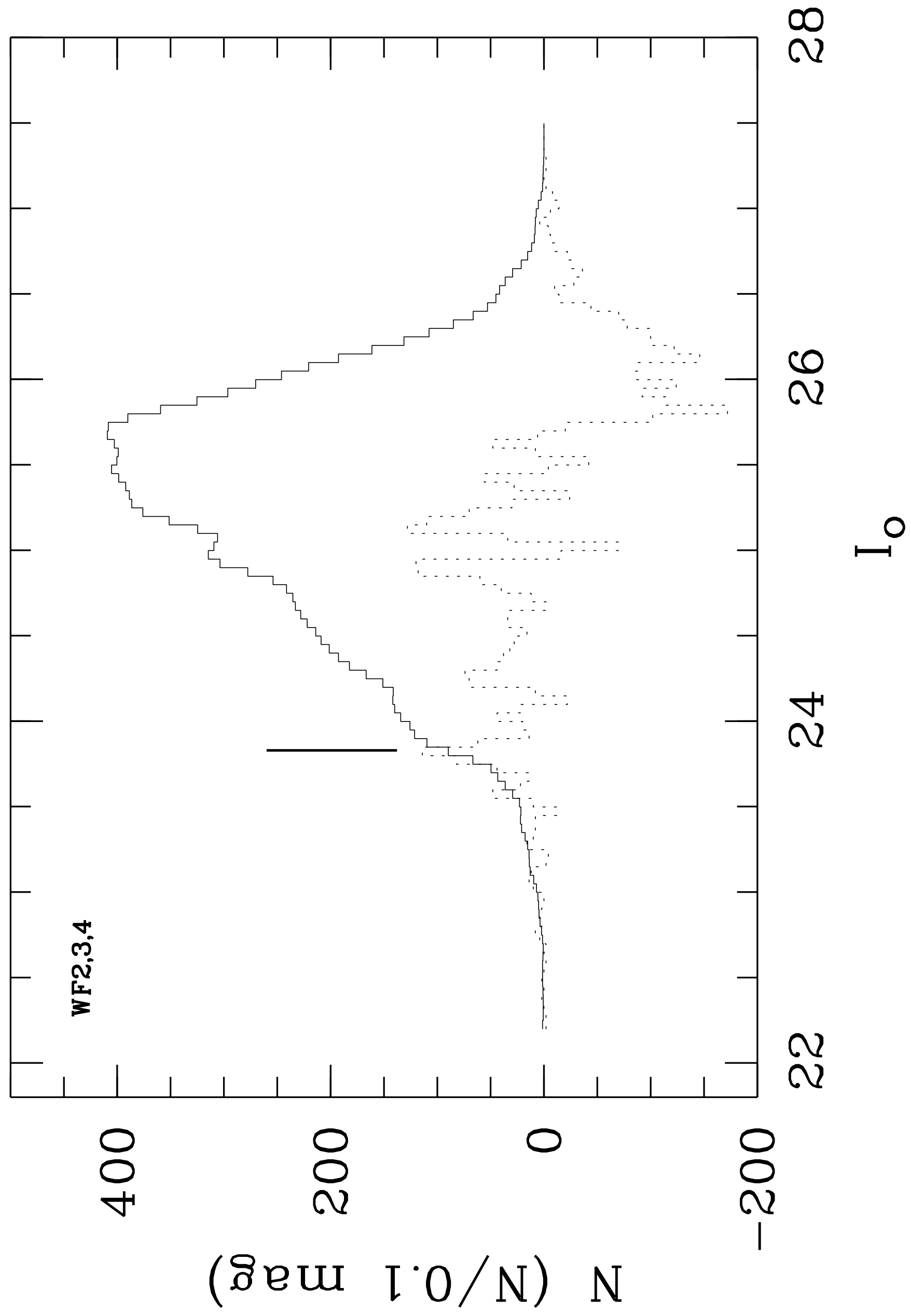

